\title{
A Methodology for Expedient Analysis of the Impact of Disorder in Periodic Waveguides
}

\author{
Juan S. Ochoa and Andreas C. Cangellaris \\ University of Illinois at Urbana-Champaign, Urbana, Illinois, 61801, United States
}

\begin{abstract}
An expedient methodology is presented for the predictive analysis of the impact of statistical disorder on the electromagnetic attributes of periodic waveguides. The proposed methodology makes use of ideas from the Anderson localization theory to derive closed-form expressions for the calculation of an effective exponential decay ratio that quantifies the impact of periodicity disorder on the transmission properties of the waveguide. The computational efficiency of the proposed method over Monte Carlo based alternatives is demonstrated through a specific example involving a periodically-loaded parallel plate waveguide.
\end{abstract}

Index Terms-Localization, disorder, periodic structure, finite element method, Monte Carlo analysis.

\section{INTRODUCTION}

Periodic waveguides are commonly used for a variety of filtering and other types of electromagnetic signal processing applications [1]. Although these structures are intended to be ideally periodic, manufacturing-induced variability results in random alterations of the geometry and material properties of the structure, which, in turn, result in degradation of its intended transmission attributes. While Monte Carlo methods are the natural candidates for the quantitative assessment of the impact of such variability on the electromagnetic attributes of the structure, an alternative, more efficient approach is possible and is proposed in this paper. This approach, which makes use of the Anderson localization theory [2], leads to the computationally-efficient calculation of an average exponential decay per unit cell for the transmitted wave, which is also known in the solid state physics literature as localization factor. As demonstrated through its application to a variety of periodic dynamic systems (see, for example, [3], [4], and [5] for applications in the structural dynamics community), this average exponential decay has been shown to provide for an accurate quantitative measure of the disorder-induced degradation of the response attributes of the periodic structure.

It is shown in this paper that Anderson localization theory offers a computationally expedient way for the computer-aided quantitative analysis of the impact of statistical variability in the geometric and material attributes of electromagnetic periodic waveguides.

The technique has been applied to analyze the effects of the disorder in transmission-line based metamaterial structures in [6] where only lumped elements and transmission-lines based structures have been considered. Whereas in this paper, the theory is adopted to investigate the effect of periodicity disorder of a structure that does not lend itself to modeling in terms of lumped circuit elements.

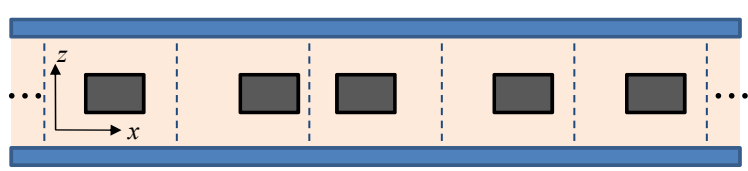

(a)

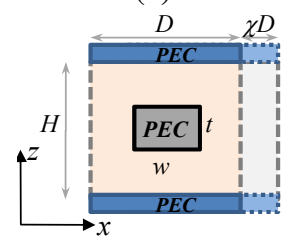

(b)

Fig. 1. (a) Longitudinal cross section of a periodic parallel-plate waveguide. (b) Perturbed unit cell

\section{THEORY}

To fix ideas, consider the case of the $y$-invariant, parallelplate waveguide structure, of longitudinal section as depicted in Fig. 1. With all conductors assumed to be perfect, the structure is operated at the fundamental (TEM) mode with wave propagation along $x$. The intended periodic loading with $y$-directed conducting strips of rectangular cross section would result, under ideal conditions of perfect periodicity, in a band-stop filter structure. However, in the presence of statistical variability in the longitudinal position of each wire, such ideal behavior is degraded. Without loss of generality, and as depicted in the figure, we choose to describe this variability in terms of a single random variable $\chi$ of zero mean value and known standard deviation $\sigma$. The extension of the proposed method to the more general case, where variability is described in terms of $m$ independent random variables, is briefly discussed in the conclusion.

In the case of an ideal structure, the transmission properties can be computed through the application of a Floquet analysis-based finite element model [7]. Through a standard Galerkin process, the finite element approximation of the vector Helmholtz equation for the electric field over the unit cell of the periodic results in a linear system of the form,

$$
\left[M_{o 1}\right] \mathbf{x}^{r}=\left[M_{o 2}\right] \mathbf{x}^{l}
$$

where $\left[M_{o 1}\right],\left[M_{o 2}\right] \in \mathbb{C}^{N \times N}$ and the entries of vectors $\mathbf{x}^{r}, \mathbf{x}^{l} \in \mathbb{C}^{N \times 1}$ are the weights of the expansion functions used to expand the electric and magnetic fields on the left and 
right walls of the unit cell, respectively. Imposing the Floquet periodic boundary condition, $\mathbf{x}^{r}=e^{-\gamma_{0} D} \mathbf{x}^{l}$, results in the linear eigenvalue problem

$$
e^{-\gamma_{0} D}\left[M_{o 1}\right] \mathbf{x}^{l}=\left[M_{o 2}\right] \mathbf{x}^{l} .
$$

The solution to (2) yields a set of $N$ eigenvalues and their respective eigenvectors. With the bandwidth of interest of the electromagnetic analysis limited to TEM mode propagation only, the eigenmodes of interest are the ones for the TEM leftand right-propagating waves. Their eigenvectors are sorted in the matrix $\left[V_{o}\right] \in \mathbb{C}^{N \times 2}$.

Consider, next, a small disorder in the position of the wire in the $n$-th cell, described in terms of a statistical variability in the width of the cell as follows,

$$
D(n)=D(1+\chi(n)) .
$$

The random variable $\chi(n)$ follows a given probability distribution, $p(\chi)$, with zero mean and standard deviation $\sigma$. It is assumed that variables $\chi(n)$, one for each cell that exhibits disorder, are independent and identically distributed. In a manner similar to the case of the ideal unit cell, the finite element model describing propagation through the $n$-th cell is of the form

$$
\left[M_{n 1}\right]\left[V_{o}\right] \mathbf{y}^{r}=\left[M_{n 2}\right]\left[V_{o}\right] \mathbf{y}^{l} .
$$

In the above equation, use was made of the fact that the vectors $\mathbf{x}^{l, r}$ at the left and right ends of the $n$-th cell can be expressed in terms of the eigenvectors of the fundamental eigenmodes $\mathbf{x}^{l, r}=\left[V_{o}\right] \mathbf{y}^{l, r}$, where $\mathbf{y} \in \mathbb{C}^{2 \times 1}$. Multiplying both sides of (4) on the left by $\left[V_{o}\right]^{H}\left[M_{n 1}\right]^{-1}$ yields

$$
\mathbf{y}^{r}=\left[V_{o}\right]^{H}\left[M_{n 1}\right]^{-1}\left[M_{n 2}\right]\left[V_{o}\right] \mathbf{y}^{l}=[T(n)] \mathbf{y}^{l} .
$$

Clearly, for the non-perturbed unit cell the above equation is of the form

$$
\mathbf{y}^{r}=\left[\begin{array}{cc}
e^{-\gamma_{0} D} & 0 \\
0 & e^{\gamma_{0} D}
\end{array}\right] \mathbf{y}^{l}=\left[T_{o}\right] \mathbf{y}^{l},
$$

Next, the assumption is made that the transmission matrix $[T(n)]$ of the $n$th perturbed cell can be expressed as the product of the ideal transmission matrix, $\left[T_{o}\right]$ and a perturbation matrix, $[Q(n)][5]$,

$$
[T(n)]=\left[T_{o}\right][Q(n)] .
$$

With these matrices defined, standard microwave network analysis techniques are used to calculate the transmission characteristics of a structure consisting of the cascade of $N$ cells exhibiting disorder. Under the assumption that cells with index $n>N$ exhibit no disorder, such an analysis yields an effective propagation factor per cell that quantifies the transmission attributes of the structure and is defined as follows [5]

$$
\gamma D=\lim _{N \rightarrow \infty} \frac{1}{N} \ln \left(t_{22}\right),
$$

where $t_{22}$ is the element $(2,2)$ of the matrix $\left[T_{T}\right]$ that represents the overall transmission of a wave traveling from left to right from the input of perturbed cell 1 to the output of perturbed cell $N$. While standard Monte Carlo analysis may be used for the calculation of the propagation factor in the presence of statistical variability, closed-form expressions for its calculation are possible under the assumption of small, moderate, and even strong reflections between adjacent cells, as detailed in [5]. In particular, for the case of small reflections it is,

$$
\gamma D=\gamma_{o} D-\int \ln \left(q_{22}(\chi)\right) p(\chi) d \chi
$$

For the case of moderate reflections, the closed-form expression becomes,

$$
\begin{gathered}
\gamma D=\gamma_{o} D-\int \ln \left(q_{22}(\chi)\right) p(\chi) d \chi+ \\
\iint \ln \left(1+\frac{\lambda^{2} q_{12}\left(\chi_{2}\right) q_{21}\left(\chi_{1}\right)}{q_{22}\left(\chi_{2}\right) q_{22}\left(\chi_{1}\right)}\right) p\left(\chi_{1}\right) p\left(\chi_{2}\right) d \chi_{1} d \chi_{2} .
\end{gathered}
$$

In the above equations, $q_{i j}(i, j=1,2)$ denote the elements of the perturbation matrix $[Q(n)]$.

In the case of small reflections, the impact of multiple reflections between adjacent cells is assumed negligible. In this case, the integration in (9) is carried out in the random domain of $\chi$, and it is done by using a quadrature rule which allows us to approximate the integral in terms of a simple weighted sum of $R$ function evaluations.

For the case of moderate reflections, reflections between adjacent cells are taken into consideration. Thus, two integration variables $\chi_{1}$ and $\chi_{2}$ are present in (10), associated with unit cells $n$ and $n-1$, and contributing the impact of the inter-cell reflection to the effective propagation factor. Since perturbation in every unit cell is assumed to be described by statistics independent from the perturbation in other cells, these two variables correspond to the same random variable $\chi$ and share the same distribution. Therefore, for the integration although a two-dimensional quadrature rule is involved on a tensor grid that involves the points of the one-dimensional quadrature along each dimension, the matrix $[Q]$ needs to be computed for the same values of the one-dimensional quadrature rule. Thus, the number of finite element solutions needed for both the case of small reflections and the case of moderate reflections is the same, $R$.

With regards to the validation of the aforementioned expressions for the attenuation constant per cell, a straightforward approach is offered through the approximation of equation (8) by the mean value of $\ln \left(t_{22}\right)$, with the matrix $\left[T_{T}\right]$ computed for a finite number $N$ of perturbed cells. The pertinent integral is,

$$
\gamma D=\int d \chi \ln \left(t_{22}(\chi)\right) p(\chi),
$$

where $\chi$ is a vector containing random variables $\chi_{i}, i=$ $1,2, \ldots, N$, that quantify the disorder in each unit cell of a chain of $N$ unit cells and $p(\chi)$, their joint probability 
distribution. Instead of a brute-force Monte Carlo scheme, an efficient multivariate quadrature rule, based on the sparse Smolyak algorithm is employed for the numerical calculation of (11) [8]. In this manner, the approximation of (11) through the weighted sum of function evaluations,

$$
\gamma D \approx \sum_{r=1}^{R} \ln \left(t_{22}\left(\boldsymbol{\chi}_{r}\right)\right) p\left(\boldsymbol{\chi}_{r}\right) w_{r},
$$

involves a number of nodes, $R$, over the random space that is significantly smaller than the one involved in a tensor product grid and is determined by a parameter called accuracy level. The details on the calculation of the Smolyak grid nodes, $\chi_{r}$, and weights, $w_{r}$, for a given level of accuracy can be found in [9].

\section{Numerical Results}

The real part of $\gamma D$ represents the exponential decay per unit cell that the wave suffers as it travels trough the disordered structure. For its calculation using (9) and (10), a one-dimensional quadrature rule with Smolyak accuracy of level 3 was utilized. Thus, the finite element solver used to compute matrices $[T]$ for a single unit cell, needs to be run only three times for each frequency. This should be contrasted to the calculation of (12) with $N=4$, where the finite element solver for the 4-cell disordered geometry had to be run 33 times for each frequency.

Referring to the geometry depicted in Fig. 1, a structure with $H / D=1$ and $w=t=0.2 D$ was analyzed. A Gaussian random variable with zero mean and standard deviations of 0.1 and 0.15 was employed to quantify the cell length disorder. Figure 2 depicts the calculated decay per unit cell. In particular, a comparison is offered of the ideal case, with zero attenuation in the pass band, with results obtained using the expressions (9) and (10), as well as from the calculation of (12) for $N=4$. As expected, the case with larger disorder, described in terms of a larger standard deviation, results in higher attenuation in the pass band. It is also clear from Fig. 2 that the computed attenuation using (9) for the case of small reflections is in very good agreement with the results from the more expensive calculation using (12), especially for frequencies to the left of the stop-band. The result obtained from (10) agrees very well with the result from (12) for frequencies on either side of the stop-band. This suggests that the proposed approach is a good candidate for the computational efficient analysis of the impact of statistical disorder on the transmission properties of periodic electromagnetic waveguides.

\section{CONCLUSIONS}

In summary, a computationally efficient statistical analysis framework has been demonstrated for the quantification of the impact of random disorder on the transmission properties of periodic waveguides. Through the use of ideas from the Anderson localization theory, the proposed model relies on the numerical modeling of the unit cell of periodic structure, within which disorder is represented in terms of an appropriate set of independent random variables, to develop closed-form

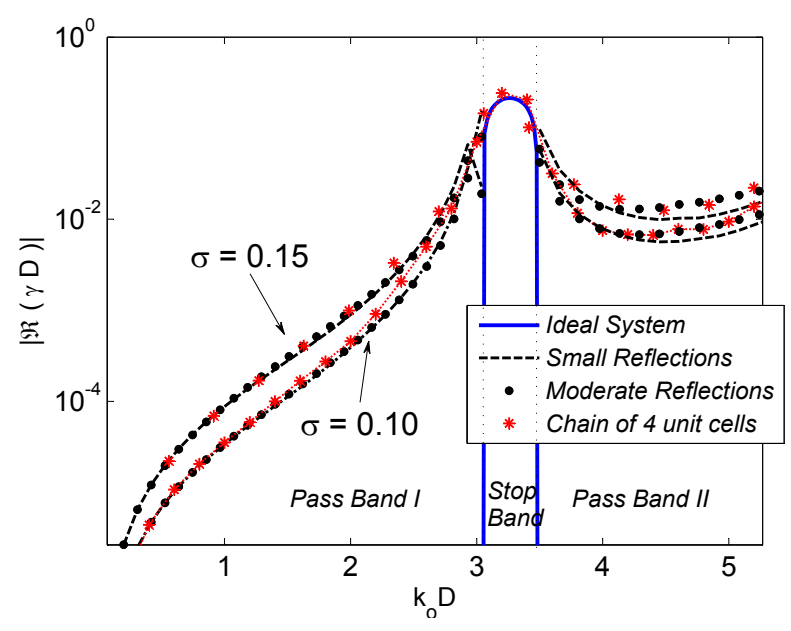

Fig. 2. Localization factor of disordered periodic array of wires. For standard deviations $\sigma=0.1,0.15$.

expressions for a disorder-induced attenuation factor per unit cell.

While the proposed method was demonstrated for the case of two-dimensional, periodic waveguide with disorder represented in terms of a single random variable, its extension is straightforward to three-dimensional waveguides exhibiting more complicated disorder that requires a multi-dimensional random space for its representation. Specific examples of such cases are currently under consideration and will be presented in a forthcoming publication.

\section{ACKNOWLEDGMENT}

This research was supported in part by the United States Army Research Office.

\section{REFERENCES}

[1] R. E. Collin, Foundations for Microwave Engineering. New York: McGraw-Hill, Inc., 1992.

[2] P. W. Anderson, "Absence of diffusion in certain random lattices," Physical Review, vol. 109, no. 5, pp. 1492-1505, March 1958.

[3] G. J. Kissel, "Localization and the invariant probability measure for a structural dynamic system," Modeling, Signal Processing, and Control for Smart Structures, vol. 7286, no. 1, April 2009.

[4] C. Pierre, "Weak and strong vibration localization in disordered structures: A statistical investigation," Journal of Sound and Vibration, vol. 139, no. 1 , pp. 111 - 132, May 1990.

[5] G. Cai and Y. Lin, "Localization of wave propagation in disordered periodic structures," AIAA journal, vol. 29, no. 3, pp. 450 - 456, March 1991.

[6] J. Ochoa and A. Cangellaris, "Analysis of the impact of statistical variations on transmission-line based metamaterial structures," in Proc. European Microwave Conference (EuMC 2010), September 2010, pp. $1397-1400$.

[7] Y. Zhu and A. Cangellaris, Multigrid Finite Element Methods for Electromagnetic Field Modeling. Hoboken, New Jersey: Wiley \& Sons, Inc., 2006.

[8] E. Novak and K. Ritter, "High dimensional integration of smooth functions over cubes," Numerische Mathematik, vol. 75, pp. 79-97, 1996.

[9] F. Heiss and V. Winschel, "Likelihood approximation by numerical integration on sparse grids," Journal of Econometrics, vol. 144, no. 1, pp. $62-80$, May 2008 . 